Western University

Scholarship@Western

$11-1-2017$

Disruption of Hippocampal Multisynaptic Networks by General Anesthetics.

Min-Ching Kuo

L Stan Leung

Follow this and additional works at: https://ir.lib.uwo.ca/physpharmpub

Part of the Anesthesiology Commons, Behavioral Neurobiology Commons, Medical Physiology

Commons, Pharmacy and Pharmaceutical Sciences Commons, and the Systems Neuroscience

Commons 


\title{
Disruption of Hippocampal Multisynaptic Networks by General Anesthetics
}

\author{
Min-Ching Kuo, Ph.D., L. Stan Leung, Ph.D.
}

\section{ABSTRACT}

Background: Previous studies showed that synaptic transmission is affected by general anesthetics, but an anesthetic dose response in freely moving animals has not been done. The hippocampus provides a neural network for the evaluation of isoflurane and pentobarbital on multisynaptic transmission that is relevant to memory function.

Methods: Male Long-Evans rats were implanted with multichannel and single electrodes in the hippocampus. Spontaneous local field potentials and evoked field potentials were recorded in freely behaving rats before (baseline) and after various doses of isoflurane ( 0.25 to $1.5 \%$ ) and sodium pentobarbital $(10 \mathrm{mg} / \mathrm{kg}$ intraperitoneal).

Results: Monosynaptic population excitatory postsynaptic potentials at the basal and apical dendrites of CA1 were significantly decreased at greater than or equal to $0.25 \%(n=4)$ and greater than or equal to $1.0 \%(n=6)$ isoflurane, respectively. The perforant path evoked multisynaptic response at CA1 was decreased by $-50 \%$ at greater than or equal to $0.25 \%$ isoflurane $(n=5)$. A decreased population excitatory postsynaptic potential was accompanied by increased paired-pulse facilitation. Population spike amplitude in relation to apical dendritic population excitatory postsynaptic potential was not significantly altered by isoflurane. Spontaneous hippocampal local field potential at 0.8 to $300 \mathrm{~Hz}$ was dose-dependently suppressed by isoflurane $(n=6)$, with local field potential power in the 50- to $150-\mathrm{Hz}$ band showing the highest decrease with isoflurane dose, commensurate with the decrease in trisynaptic CA1 response. Low-dose pentobarbital $(n=7)$ administration decreased the perforant path evoked trisynaptic CA1 response and hippocampal local field potentials at 78 to $125 \mathrm{~Hz}$.

Conclusions: Hippocampal networks are sensitive to low doses of isoflurane and pentobarbital, possibly through both glutamatergic and $\gamma$-aminobutyric acid-mediated transmission. Network disruption could help explain the impairment of hippocampal-dependent cognitive functions with low-dose anesthetic. (ANESTHesıology 2017; 127:838-51)

G ENERAL anesthetics affect brain activity and function at different doses. ${ }^{1,2}$ The dose required for surgical anesthesia, which suppresses incision pain and movements, is higher than that for loss of consciousness, validated by a lack of response to verbal commands in humans. Lower doses of general anesthetic disrupt cognitive function, with different types of memory showing differential sensitivity. ${ }^{3,4}$ The mechanisms whereby general anesthetics affect behavioral and neural functions are not completely known. ${ }^{1,2}$

Many studies of general anesthetics have focused on in vitro brain preparations, and advances have been made on the effect of general anesthetics on ion channels and receptors. ${ }^{5}$ Early work of Richards and White ${ }^{6}$ reported that volatile anesthetic decreased excitatory synaptic transmission in the dentate gyrus (DG) of the hippocampus in vitro, at doses less than 1 minimal alveolar concentration (MAC). Volatile anesthetics less than $1 \mathrm{MAC}$ were also shown to decrease non-N-methyl-D-aspartate receptor-mediated glutamatergic transmission in the hippocampal CA1 area. ${ }^{7-11}$ Glutamatergic neurons have been shown to be susceptible to metabolic depletion by a volatile anesthetic. ${ }^{12} \gamma$-Aminobutyric

\section{What We Already Know about This Topic}

- Isoflurane produces amnesia at concentrations lower than those required for surgical anesthesia. The hippocampus is central to memory and learning, and in vitro work has shown that isoflurane can disrupt network function in the hippocampus.

- The effect of isoflurane on hippocampal network function, particularly at low doses that produce amnesia, has not been fully characterized in freely moving animals in vivo.

\section{What This Article Tells Us That Is New}

- Monosynaptic and multisynaptic excitatory postsynaptic potentials, and spontaneous local field potentials, in the CA1 sector of the hippocampus were significantly suppressed by isoflurane at low doses and in a dose-dependent manner.

- The results, which confirm previous in vitro work, are consistent with the premise that hippocampal network disruption may underlie isoflurane-induced, hippocampaldependent cognitive function.

acid-mediated (GABAergic) inhibition in vitro was sensitive to low doses of volatile anesthetics. ${ }^{13-15}$

The relation between brain activity and behavioral functions is best studied in freely moving animals in vivo. This

Submitted for publication March 1, 2017. Accepted for publication August 1, 2017. From the Department of Physiology and Pharmacology (M.-C.K.) and the Department of Physiology and Pharmacology and Program in Neuroscience (L.S.L.), The University of Western Ontario, London, Ontario, Canada. 

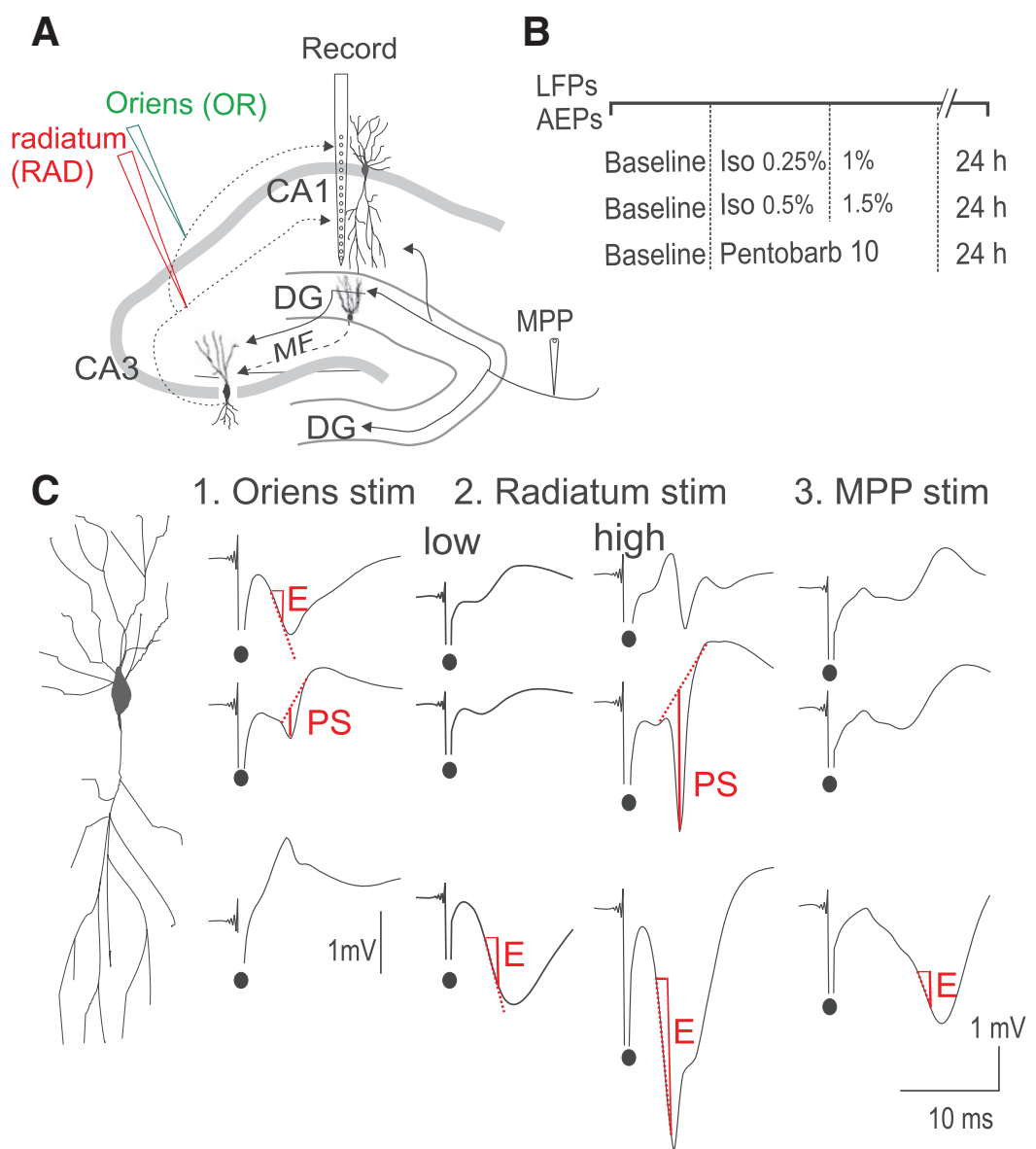

Fig. 1. Stimulation and recording of monosynaptic and multisynaptic field potentials in hippocampal CA1 area. $(A)$ Schematic of hippocampal circuit. Medial perforant path (MPP) projects to the dendrites of granule cells in the dentate gyrus (DG), which excite CA3 through mossy fibers (MF). CA3 pyramidal cells project Schaffer collaterals to the basal and apical dendrites of CA1 pyramidal cells, respectively excited by stimulation of stratum (str.) oriens (OR) and stratum radiatum (RAD), and recorded by a 16-channel silicon recording probe in CA1. (B) Time schedule of recordings of spontaneous local field potentials (LFPs) and average evoked field potentials (AEPs), each experiment with baseline, isoflurane (Iso) at two doses or pentobarbital $10 \mathrm{mg} / \mathrm{kg}$ intraperitoneal, and then $24 \mathrm{~h}$ later. (C) AEPs shown at three layers in CA1: basal dendritic, somatic (stratum pyramidale), and mid-apical dendritic layers, after stimulation (stim) of str. oriens (C1), radiatum (C2), and MPP (C3). Synaptic excitation (E) was analyzed in the layer excited as the negative slope over a duration of $1 \mathrm{~ms}$. A population spike (PS), indicating synchronous firing of neuronal units, was analyzed at str. pyramidale after a high stimulus intensity. MPP stimulation resulted in late (10 to $15 \mathrm{~ms})$ trisynaptic excitation of the CA1 apical dendrites (C3). Solid circles indicate shock artifacts.

approach can elucidate the brain mechanisms underlying cognitive impairment and loss of consciousness after low doses of general anesthetic. The in vivo brain offers intact neural networks that are not altered by in vitro slicing or culturing. To our knowledge, other than a few exceptions, ${ }^{16}$ the effects of low-dose general anesthetic on neural activity in vivo have not been studied.

We focused on the hippocampal cortex, which is particularly well structured for network studies of neural interactions. ${ }^{17,18}$ The hippocampus is also important for contextual fear conditioning, ${ }^{19}$ which was disrupted by isoflurane of $0.25 \mathrm{MAC}^{3}$ and MAC for isoflurane was $-1.4 \%$ in rats. ${ }^{20}$ A multisynaptic pathway from the entorhinal cortex (EC) to the DG, CA3, and CA1 areas of the hippocampus (fig. 1A $)^{17,18}$ has been implicated as a main neural circuit for declarative memory in humans. ${ }^{21}$ Each of the excitatory synapse at the EC-DG-CA3-CA1 circuit is glutamatergic, but GABAergic interneurons provide inhibition at somatic, dendritic, and presynaptic sites. ${ }^{22}$ There is controversy over whether isoflurane affects the excitatory ${ }^{23}$ or inhibitory transmission ${ }^{24}$ in the hippocampus of intact animals, and electrophysiologic responses to low doses of isoflurane (less than $0.35 \%$ ) have not been studied.

We hypothesize that the multisynaptic networks in the hippocampus in vivo are sensitive to isoflurane, even at low doses (less than $0.5 \%$ ). The first objective was to compare multisynaptic (EC to CA1) transmission with monosynaptic (CA3 to CA1) excitation at the basal or apical dendrites of the CA1 pyramidal cells. A second objective was to study networks by spontaneous local field potentials (LFPs), 
and $\Delta, \Theta$, and $\gamma$ frequencies in the hippocampal LFPs are known to be generated by extrinsic and intrinsic neural circuitry. ${ }^{25-30}$ For comparison to low-dose isoflurane responses, the effects of a single subanesthetic dose of sodium pentobarbital ( $10 \mathrm{mg} / \mathrm{kg}$ intraperitoneal) on hippocampal synaptic transmission and spontaneous LFPs were also studied.

\section{Materials and Methods}

\section{Animals}

Adult male Long-Evans rats (Charles River, Canada) were kept in a temperature-controlled room under $12: 12 \mathrm{~h}$ light-dark cycle (lights on/off at 7:00/19:00 h) with food and water available ad libitum. All experiments were conducted between 9:00 and 17:00 h in accordance with the guidelines established by the Canadian Council on Animal Care (Ottawa, Canada) and approved by the local Animal Use Committee at The University of Western Ontario (London, Ontario, Canada).

\section{Surgery and Recording}

Male Long-Evans rats (250 to $350 \mathrm{~g}$ ) were handled and habituated to a recording chamber $(30 \times 30 \times 30 \mathrm{~cm}$ Plexiglas cage $)$ for at least 3 days before surgery. Under sodium pentobarbital anesthesia $(60 \mathrm{mg} / \mathrm{kg}$ intraperitoneal, supplemented as needed), animals were placed in a stereotaxic apparatus. Body temperature was maintained between 35 to $37^{\circ} \mathrm{C}$ with a rectal thermometer monitoring system. In seven rats, a 16-channel silicon probe $(50-\mu \mathrm{m}$ interelectrode distance; NeuroNexus, USA) was inserted to span all layers of the right dorsal hippocampal CA1 area (P4.6, L3.2, V3.3; atlas of Paxinos and Watson $\left.{ }^{31}\right)$. Teflon-coated steel wires of $125-\mu \mathrm{m}$ diameter were used as stimulating electrodes, placed at (1) right medial perforant path (MPP; P8.0, L4.4, V3.5), (2) stratum radiatum (RAD), and (3) stratum oriens (OR) of CA1 bilaterally (P4.6, L3.0, V3.0; P3.2, L2.0, V3.0). All recordings in CA1 were made with a 16-channel probe, unless otherwise stated. In four additional rats, recordings were done with Teflon-coated stainless steel wires in the dentate gyrus (P3.8, L 2.7, V4), and two of the rats also had stimulus electrodes in OR and recording electrodes in CA1. Optimal placement of the stimulating and recording electrodes were made by electrophysiologic criteria. ${ }^{32,33} \mathrm{~A}$ recording screw was located at the frontal cortex (A1.0, L1.0) and other ground and reference screws were placed in the skull over the cerebellum. All electrodes were fixed by creating a head cap made of dental cement. A stimulus isolation unit (PSIU6 connected to Grass S44 stimulator, Astro-Med, Inc., USA) provided constant current stimulus pulses (0.2-ms duration, less than or equal to $400 \mu \mathrm{A}$ ), delivered cathodally to a stimulus electrode, with a screw over the cerebellum serving as anode.

\section{Experimental Procedures}

Recording sessions commenced at least 7 days after surgery, after further habituation in the Plexiglas recording chamber. Signals recorded by a silicon probe were fed through a headstage to a commutator, and then amplified and filtered ( 1 to $10 \mathrm{kHz}$ ) by a Medusa preamplifier and RA16 base station (Tucker-Davis Technologies, USA) before digitization (typically at $6 \mathrm{kHz}$ ) by a custom-made program.

A rat underwent two or more recording sessions with isoflurane anesthesia, separated by at least 5 days (fig. 1B). Isoflurane in $100 \%$ oxygen from a vaporizer was delivered by tubing through a small outlet to the bottom of the chamber, and allowed to flow out through a small hole at the top of the recording chamber for continuous monitoring by means of an infrared gas analyzer (Ohmeda RGM5250, USA). For the first session, recordings were first made during baseline before anesthetic administration, followed by isoflurane administration at $0.25 \%$ and then $1 \%$, before returning to room air (0\%). Follow-up recordings were made at $24 \mathrm{~h}$ after isoflurane exposure. Recordings started at less than $20 \mathrm{~min}$ after each isoflurane concentration change, when the gas analyzer indicated a stable isoflurane concentration. In order to keep the rat near its normal body temperature, a heating pad maintained at $36^{\circ} \mathrm{C}$ was placed underneath the recording chamber. For the next session 5 or more days later, after baseline recording, isoflurane was delivered at $0.5 \%$, and then at $1.5 \%$. For subsequent sessions, some rats were given $0.375 \%$ isoflurane, or a previous isoflurane dose was repeated. No attempts were made to randomize the doses or to blind the experimenter, since the behavioral effects of high-dose isoflurane were clearly discernible. In another session, sodium pentobarbital $(10 \mathrm{mg} / \mathrm{kgintraperitoneal})$ was injected, with recordings done during baseline (before pentobarbital) and at $15 \mathrm{~min}, 1 \mathrm{~h}$, and $24 \mathrm{~h}$ after pentobarbital.

LFPs were recorded for minutes during different behavioral states before and after different isoflurane doses. Immobility state was defined when the rat spontaneously showed no gross head and body movements. If not administered with an anesthetic, the rat had to be awake and typically held its head up. Walking state was operationally defined to include horizontal locomotion, rearing, and large head and body movements. Rapid eye movement (REM) sleep was defined when the rat was immobile while a regular hippocampal $\Theta$ rhythm $(3$ to $10 \mathrm{~Hz})$ was detected. Hippocampal average evoked potentials ( $\mathrm{n}=5$ sweeps) were recorded during immobility, excluding REM periods, after stimulation of $\mathrm{OR}$ and $\mathrm{RAD},{ }^{32,33}$ or $\mathrm{MPP}^{34}$ (fig. 1C). Different stimulus intensities were applied to each pathway, including two and four times the stimulus threshold, with threshold defined as the stimulus intensity that evoked a visually detectable response. Behaviors after anesthetic administration were noted, including locomotor activity, ataxia, or extended periods of inactivity. Single- or paired-pulse stimulation used for evoking field potentials did not induce detectable behaviors. At the end of the experiment, under pentobarbital $(80 \mathrm{mg} / \mathrm{kg}$ intraperitoneal) anesthesia, the rat was perfused with saline followed by $4 \%$ formaline through the heart. The brain was retrieved and subsequently, the locations of the electrodes were verified in $40-\mu \mathrm{m}$ thionin-stained brain sections. 


\section{Analysis of Data}

Population postsynaptic potential (pEPSP) and population spike (PS) were distinguished in the average evoked potentials. PS was generated by synchronously firing single neurons. ${ }^{17,32}$ Monosynaptic excitation after stimulation of OR (or RAD) was measured by the rising slope of the negative pEPSP at OR (or RAD). The maximal slope over 1-ms interval was evaluated during the negative going phase of the pEPSP (fig. 1B), at $0.5 \mathrm{~ms}$ before the onset of a PS. The latter slope measure was measured within 1 to $4 \mathrm{~ms}$ of onset, during which evoked inhibitory postsynaptic potential would be expected to be minimal. The multisynaptic excitation in CA1 was evaluated by the maximal negative slope of the apical dendritic pEPSP at 10 to $15 \mathrm{~ms}$ after MPP stimulation (fig. 1B). Paired-pulse facilitation (at 50-ms interpulse interval) was evaluated by the ratio of the pEPSP slope evoked by the second pulse (E2) to that evoked by the first pulse (E1).

For silicon probe recordings, a one-dimensional current source density (CSD) was calculated from the average evoked potentials. CSD shows the local current source and sink in a laminated structure, without the effects of volume conduction..$^{35} \operatorname{CSD}(\mathrm{z}, \mathrm{t})$, a function of depth $\mathrm{z}$ and time $\mathrm{t}$, was calculated by a second-order difference formula: ${ }^{35}$

$$
\begin{aligned}
\operatorname{CSD}(\mathrm{z}, \mathrm{t})= & \sigma[2 \Phi(\mathrm{z}, \mathrm{t})-\Phi(\mathrm{z}+2 \Delta \mathrm{z}, \mathrm{t}) \\
& -\Phi(\mathrm{z}-2 \Delta \mathrm{z}, \mathrm{t})] /(2 \Delta \mathrm{z})^{2},
\end{aligned}
$$

where $\Phi(\mathrm{z}, \mathrm{t})$ is the field potential at depth $\mathrm{z}$ and time $\mathrm{t}$, and $\Delta \mathrm{z}$ is the spacing $(50 \mu \mathrm{m})$ between adjacent electrodes on the 16-channel probe. The conductivity $\sigma$ was assumed to be constant and the CSDs were reported in units of $\mathrm{V} / \mathrm{mm}^{2}$.

CSD analysis was used to give the input-output relation between PS and excitation after RAD stimulation. For a CSD trace near the CA1 pyramidal cell layer, the amplitude of the PS sink was assessed by the vertical distance of the negative sink peak to the tangent line linking the positive peaks surrounding the negative peak (fig. 1B). The strength of synaptic excitation was evaluated by the maximal slope (1 ms interval) of the apical dendritic excitatory sink. PS (y) values were plotted against excitatory sink $(\mathrm{x})$ values for each experiment, before and after isoflurane. A second-order polynomial $\left(y=a x^{2}+b x+c\right.$, where $a, b, c$ are fitted numbers) was drawn to fit all the points during baseline. For a given $\mathrm{x}$ range, a cluster of isoflurane points consistently above the baseline curve was considered to be a significant deviation from the baseline. Wilcoxon test between baseline and isoflurane groups (minimum four per group over a fixed x range) will determine the $P$ value.

Spontaneous LFPs were sampled at $6.103 \mathrm{kHz}$, and power spectral analysis was done by custom software, as described previously. ${ }^{36}$ Artifact-free activity was selected manually. Each LFP segment consisted of 8,192 points $(1.34 \mathrm{~s})$, and one tenth of the total segment length was tapered by a cosine function. $(1-\cos (10 \pi t / T))$ where $t$ is time from each end and $T$ is the segment length. After fast Fourier transform, the spectral values were smoothed by an elliptical window decreasing at $\left[1-(f i / f m)^{2}\right]^{0.5}$, where $f i=$ deviation from the center frequency and $f m=$ half the smoothing bandwidth $=2$. Effectively, the frequency resolution is $0.745 \mathrm{~Hz}$ (reciprocal of $1.34 \mathrm{~s}$ ), and after smoothing over five frequency bins, statistically independent values were $3.73 \mathrm{~Hz}$ apart. Power from 57 to $63 \mathrm{~Hz}$ (line power) was excluded. Each power spectral value derived from one segment is estimated to have 10 degrees of freedom (df), and the average spectrum of each condition consisted of 6 to 12 segments, that is, 60 to $120 \mathrm{df}$.

Based on previous studies, the SD of the pEPSP slope (normalized to mean $=1$ ) was less than or equal to $0.122 .{ }^{33}$ For a mean pEPSP decrease of 0.2 , and a two-tailed t-distribution $(\alpha=0.05)$, a sample size $\mathrm{n} \geq 5$ predicts power greater than 75\%. GB Stat (Dynamic Microsystems, Inc., USA) and SAS 9.1.4 (SAS Institute, Inc., USA) were used for statistical evaluation. The mean $\pm S D$ are shown. The change of a neural measure (pEPSP or excitatory sink slope, LFP power at a certain frequency slope, and peak $\Theta$ frequency) with isoflurane dose was analyzed by one-way repeated measures ANOVA. Unless otherwise noted, there were no missing values in the ANOVA. If the overall repeated measures ANOVA was significant $(P<\alpha, \alpha=0.05$; two-tailed), post hoc Newman-Keuls test was applied to compare values at different isoflurane doses to the baseline; significance of each post hoc test was kept at the $P<0.05$ level.

Group differences in LFP power were assessed in two ways. First, for each condition (baseline or anesthesia), a mean ensemble power spectrum was constructed by averaging the logarithmic power at each digitized frequency for all rats. At each digitized frequency bin, statistical difference in power between two conditions was evaluated by a paired Wilcoxon test. To compensate for multiple comparisons, only significant power differences that extend over five or more frequency bins (estimated $P<0.001$ ) were shown in the plots. Second, for dose response, LFP power at selected frequency bands was averaged for each rat for all experiments of a given isoflurane dose (baseline $=$ zero dose). For each frequency band, if a one-way repeated measures ANOVA was significant $(P<0.05)$, post hoc Newman-Keuls test was used to reveal differences at a particular dose as compared to the baseline. A two-way randomized block repeated measures ANOVA was applied when the same two factors were studied in the same rats (apical dendritic responses to RAD/ MPP stimulation and isoflurane dose; peak $\Theta$ frequency in relation to behavioral state and isoflurane dose).

\section{Results}

\section{Basal and Apical Excitatory Postsynaptic Potentials in CA1}

Two different synapses from CA3 to CA1 pyramidal cells were assessed: basal dendritic excitation after stimulation of $\mathrm{OR}$ and apical dendritic excitation after stimulation of RAD 


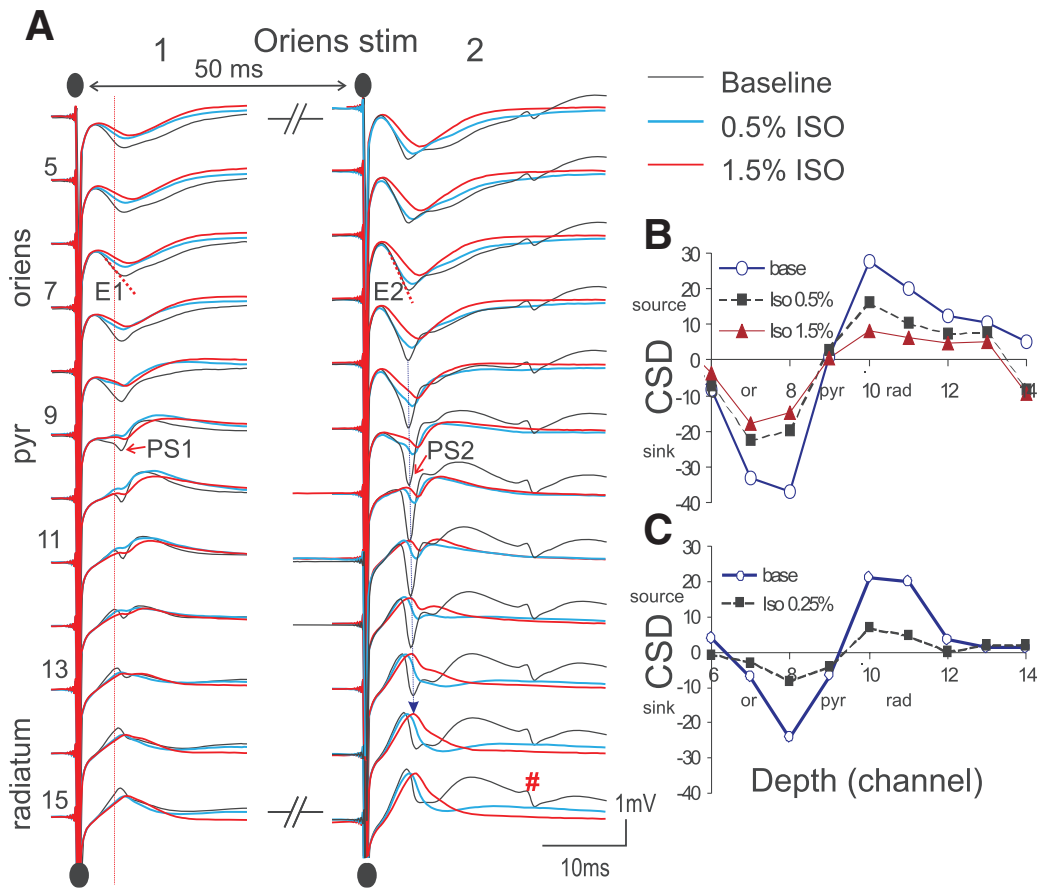

Fig. 2. Basal dendritic excitation in hippocampal CA1 after different concentrations (\% volume) of isoflurane (ISO). (A) Representative average evoked potentials in CA1 was recorded after paired-pulse stimulation ( $50 \mathrm{~ms}$ interpulse interval) of str. oriens in one rat $(\mathrm{CM} 13 ; 80 \mu \mathrm{A})$, with responses after 0.5 and $1.5 \%$ isoflurane overlaid on baseline responses. The slope of the population excitatory postsynaptic potential (pEPSP) recorded in the basal dendritic layer (str. oriens) after the second pulse (E2 in column 2) was larger than that after the first pulse (E1 in column 1). Population spikes, PS1 and PS2 (near str. pyramidale, pyr), were evoked by the first and second pulse, respectively, and PS2 is shown to propagate to str. radiatum (dotted, slanted, vertical arrow). E1 was strongly decreased by 0.5 and $1.5 \%$ isoflurane (A1), while E2 was more strongly decreased by $1.5 \%$ than $0.5 \%$ isoflurane, although PS was suppressed by $0.5 \%$ isoflurane (A2). Late ( $20 \mathrm{~ms}$ latency) potentials, interpreted as recurrent dentate gyrus excitation (\#) were abolished by 0.5 and $1.5 \%$ isoflurane (A2). (B) Depth profile of current source density (CSD, in units of $\mathrm{mV} / \mathrm{mm}^{2}$ ) at $\sim 2 \mathrm{~ms}$ after onset of excitation (vertical dotted line in part A1) during baseline (base), showing the basal dendritic sink-source pattern declined with isoflurane 0.5 and $1.5 \%$. (C) Same as $(B)$ for a different experiment on the same rat, 5 days earlier, showing $0.25 \%$ isoflurane decreased the baseline sink-source pattern.

(fig. 1). Basal dendritic pEPSPs were negative at the basal dendrites and positive at the cell layer and apical dendritic layer. A representative example showed that the slope (E1) and peak of the negative wave (pEPSP) recorded in CA1 OR evoked by first pulse of a pair of OR stimulus pulses were strongly decreased by $0.5 \%$ isoflurane (fig. 2A1). The slope of the pEPSP (E2) evoked by the second pulse was less strongly decreased by $0.5 \%$ isoflurane (fig. $2 \mathrm{~A} 2$ ). However, the population spikes evoked by the first (PS1) and second pulse (PS2) were both sensitive to $0.5 \%$ isoflurane (fig. $2 \mathrm{~A}$ ). Increase of isoflurane to $1.5 \%$ only attenuated E1 slightly more than $0.5 \%$ (fig. 2A1). CSD analysis revealed an excitatory sink (negative CSD) at the CA1 OR, accompanied by a source at $\mathrm{CA} 1$ cell layer and RAD (fig. 2B). Isoflurane (0.25\%) decreased the amplitude of the whole sink-source pattern (fig. 2C), also found for other isoflurane doses (fig. 2B).

The group data showed similar results. One-way repeated measures ANOVA showed that the normalized first-pulse pEPSP slope (E1) evoked by $2 \mathrm{x}$ threshold stimulus intensity was significantly different for six conditions (baseline, four isoflurane doses, and $24 \mathrm{~h}$ after isoflurane), as indicated by a main group effect $(\mathrm{F}[5,15]=10.3, P<0.001, \mathrm{n}=4$ rats; fig. 3). Post hoc Newman-Keuls test showed that each of the four isoflurane doses $(0.25,0.5,1.0$ and $1.5 \%)$ was significantly different from the baseline, or from $24 \mathrm{~h}$ postisoflurane, but not from each other (fig. 3). Similar results were found for E1 evoked by $4 \mathrm{x}$ threshold stimulus intensity (fig. 3). The low-dose (less than $0.5 \%$ ) isoflurane response was also confirmed in two other rats recorded by single-wire electrodes in CA1 after OR stimulation (data not shown).

The first pulse apical dendritic pEPSP (apical E1) was slightly decreased by $0.5 \%$ isoflurane and strongly attenuated by $1.5 \%$ isoflurane (fig. $4 \mathrm{~A}$ ). CSD analysis showed that the apical dendritic pEPSP was generated by a dominant sink at RAD, with source at the cell layer, and the whole source-sink pattern was suppressed by isoflurane in a dosedependent manner (fig. 4B). Repeated measures ANOVA of the group apical E1 data at $2 \mathrm{x}$ threshold stimulus intensity 

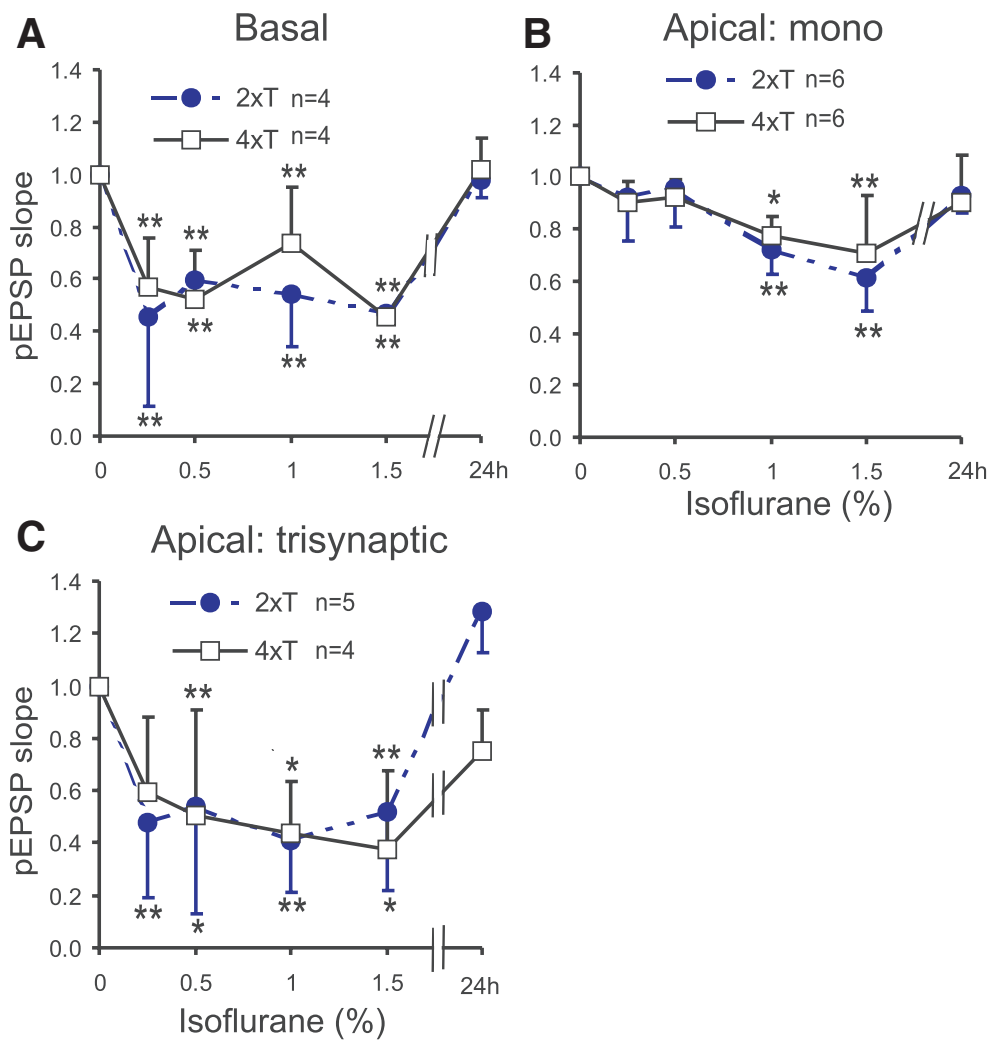

Fig. 3. Group data of normalized slope population excitatory postsynaptic potential (pEPSP) \pm SD, evoked by $2 x$ and $4 x$ threshold $(T)$ stimulation, after different doses (\% volume) of isoflurane; baseline is 0 dose, and $24 \mathrm{~h}$ was the average recorded 1 day after isoflurane administration. (A) Normalized basal dendritic pEPSP slope evoked by stimulation of stratum oriens. (B) Normalized monosynaptic apical dendritic (Apical: mono) pEPSP slope after stratum radiatum stimulation. (C) Normalized trisynaptic apical dendritic (Apical: trisynaptic) pEPSP slope after medial perforant path stimulation. All recordings were made by a multichannel silicon probe. ${ }^{\star \star} P<0.01$, ${ }^{\star} P<0.05$, difference from baseline, post hoc Newman-Keuls test, after a significant group effect in one-way repeated measures ANOVA; $n$ is the number of rats used.

confirmed an isoflurane treatment effect $(\mathrm{F}[5,25]=11.7, P$ $<0.0001, \mathrm{n}=6)$, with significant $(P<0.05)$ post hoc differences (Newman-Keuls test) only found at greater than or equal to $1 \%$ isoflurane (fig. 3). Group data of apical E1 of 4 $\mathrm{x}$ threshold stimulus intensity also showed a significant isoflurane treatment effect with post hoc differences at greater than or equal to $1 \%$ isoflurane (fig. 3).

\section{Medial Perforant Path Evoked Multisynaptic Pathway}

A long-latency CA1 multisynaptic response (triE1), manifested as a negative wave at 10 to $15 \mathrm{~ms}$ in CA1 RAD after MPP first-pulse stimulation (fig. 4C1), was significantly attenuated to $-50 \%$ of the baseline by isoflurane dose of greater than or equal to $0.25 \%$ (figs. 3 and $4 \mathrm{C} 1$ ). After paired-pulse MPP stimulation, the second pulse multisynaptic response (triE2) was less sensitive to isoflurane than triE1, while the PS after the second pulse (triPS in fig. $4 \mathrm{C} 2$ ) was strongly attenuated by $0.5 \%$ isoflurane. One-way repeated measures ANOVA of the normalized triE1 group data revealed a significant isoflurane effect $(F[5,20]=11.7$, $P<0.0001, \mathrm{n}=5)$ at $2 \mathrm{x}$ threshold stimulus intensity. Significant isoflurane effect was also found at $4 \mathrm{x}$ threshold stimulus intensity, with less robust post hoc differences (fig. 3).
The effect of isoflurane on the mulitsynaptic response (triE1) was different from that on the apical dendritic response (apical E1) evoked by RAD stimulation. In four rats that had both triE1 and apical E1 recorded, randomized block ANOVA showed a significant group $\mathrm{x}$ dose effect $(\mathrm{F}[5,15]=13.3, P<0.0001)$ without a main group effect $(\mathrm{F}[1,3]=2.55, P>0.2)$, and post hoc comparisons indicated significant differences only at isoflurane doses of 0.25 to $0.5 \%$.

The multisynaptic CA1 response resulted from activation of the DG, which then activated CA3. The PS evoked by MPP in or near the DG was not significantly changed by isoflurane dose of 0.25 to $0.5 \%$ ( $\mathrm{n}=5$; data not shown), suggesting that transmission in CA3 or CA1, and not from MPP to the DG, was the cause of the depression at low-dose isoflurane. Synaptic responses in CA3 were not recorded.

Strong stimulation of $\mathrm{CA} 3$ was shown to induce recurrent excitation of the DG through a CA3-CA1-EC-DG pathway. ${ }^{37} \mathrm{~A}$ recurrent DG excitation was observed in CA1 after stimulation of OR or RAD, typically after paired pulses (\# in fig. 2A2). The late negative wave at $-20 \mathrm{~ms}$ latency after strong OR stimulation was abolished by greater than or equal to $0.25 \%$ isoflurane (fig. 2A2), which was confirmed in five rats. 
Radiatum stim

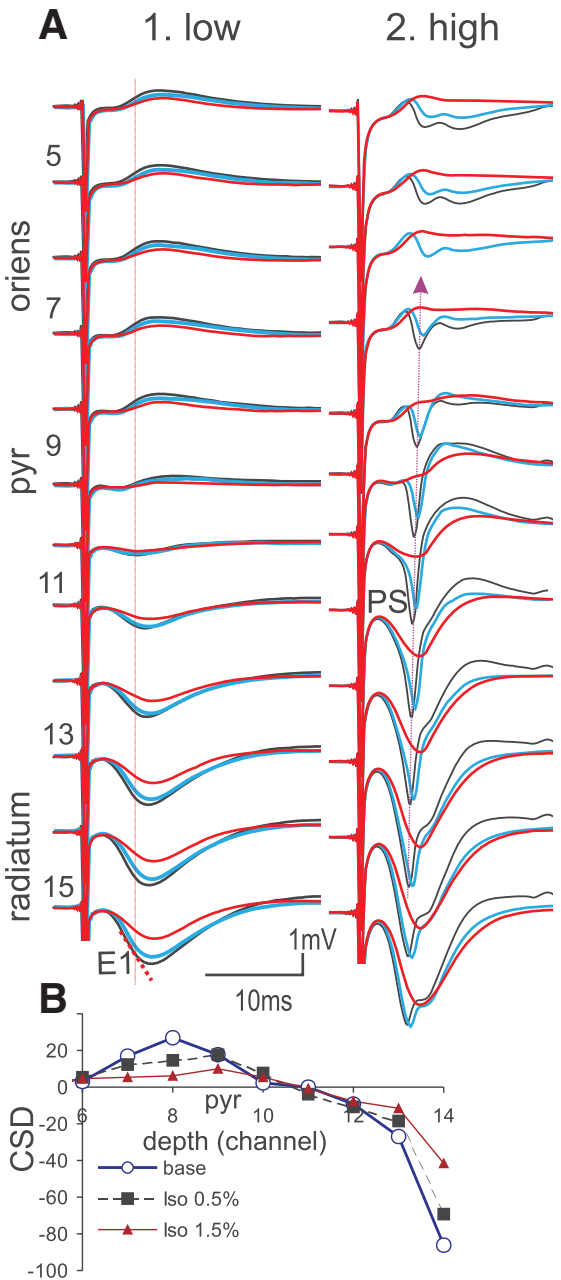

MPP stim to CA1

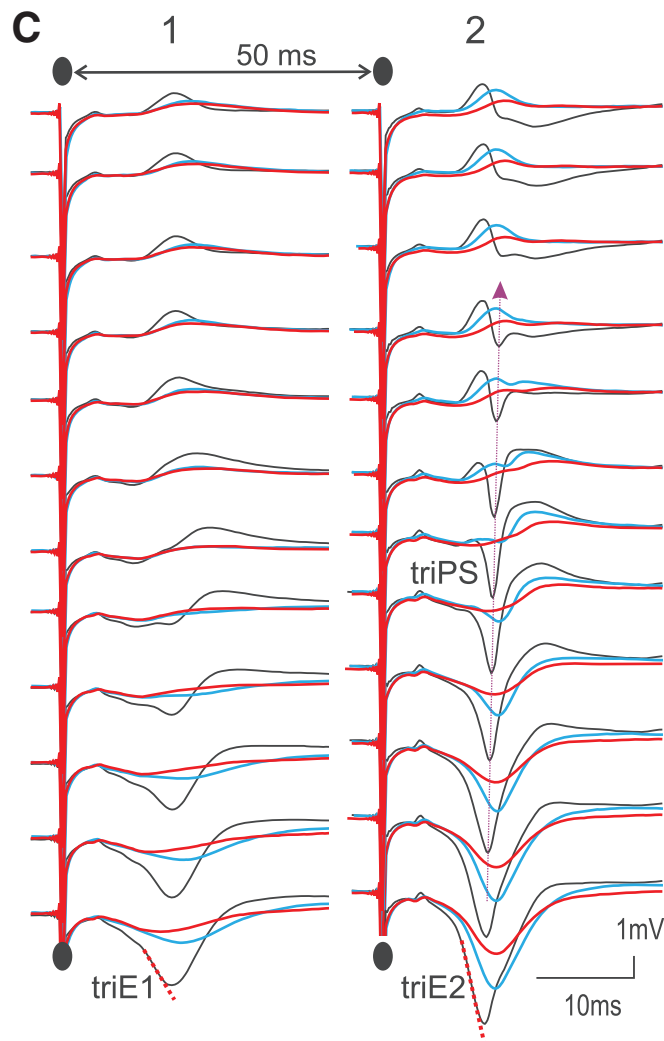

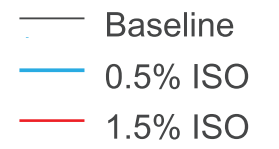

Fig. 4. Representative monosynaptic and mutlisynaptic apical dendritic excitation in hippocampal CA1 after different concentrations (\% volume) of isoflurane (ISO). (A) Average evoked potentials in CA1 was recorded after low- $(40 \mu \mathrm{A})$ and high-intensity (120 $\mu \mathrm{A})$ stimulation of str. radiatum in one rat (CM13), with responses after 0.5 and $1.5 \%$ isoflurane overlaid on baseline responses. High-intensity stimulation resulting in population spike (PS) near str. pyramidale (pyr) in column; the PS was inferred to propagate to str. oriens (dotted, slanted, vertical arrow). The population excitatory postsynaptic potential (pEPSP), measured by the slope E1, and PS were more strongly decreased by $1.5 \%$ than $0.5 \%$ isoflurane. $(B)$ Depth profile of current source density $(C S D$, in units of $\mathrm{mV} / \mathrm{mm}^{2}$; sink is negative) at the time of the dotted vertical line in part A, showing an apical dendritic excitatory sink, and its decrease with 0.5 and $1.5 \%$ isoflurane. (C) Average evoked potentials in CA1 after $120 \mu \mathrm{A}$ paired-pulse (50-ms interval) stimulation of the medial perforant path (MPP), and trisynaptic ( 10 to $15 \mathrm{~ms}$ latency) pEPSP, measured as slopes triE1 and triE2 after the first and second pulse, respectively. The trisynaptic response was suppressed by isoflurane in a dose-dependent manner.

\section{Paired-pulse Facilitation and Dose-response of Different Excitation}

During baseline, paired-pulse facilitation (PPF) of the pEPSPs at 50-ms interpulse interval was large for stimulus intensities that did not evoke a first-pulse PS, as shown for $2 \times$ threshold basal (OR stimulation), apical-monosynaptic (RAD stimulation), and apical-trisynaptic (MPP stimulation) excitation (fig. 5A). For $2 \mathrm{x}$ threshold RAD stimulation, a significant increase in PPF was found at greater than or equal to $1 \%$ but not at less than $1 \%$ isoflurane (fig. $5 \mathrm{~B}$; repeated measures ANOVA, $\mathrm{n}=7$ pathways in five rats). At $2 \mathrm{x}$ threshold stimulus intensity, PPF of the pEPSPs after OR stimulation was typically higher than that after RAD stimulation, and greater than or equal to $0.25 \%$ isoflurane further increased the PPF (fig. 5, B and C). Similarly, the late, trisynaptic response in CA1 after MPP stimulation showed a high PPF that greatly increased after greater than or equal to $0.25 \%$ isoflurane (fig. $5, \mathrm{~B}$ and C).

\section{Population Spike Amplitude and Latency Were Affected by Isoflurane}

PS in relation to synaptic excitation was analyzed for RAD stimulation only. At a fixed stimulus intensity, the PS amplitude usually decreased after isoflurane, but the change was 
A ALL 1

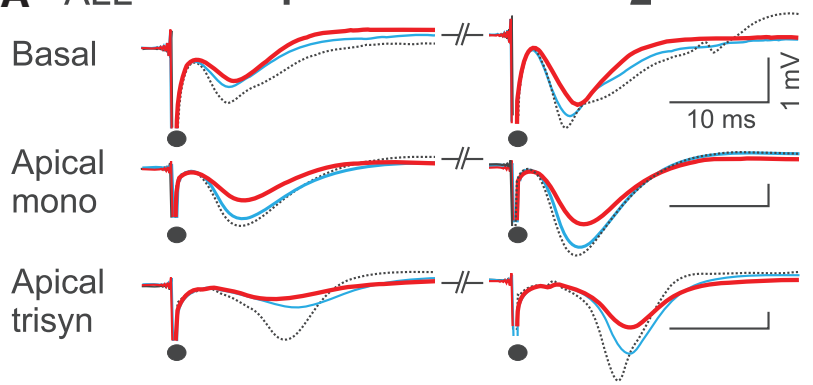

B ISO $0.5 \%$ scaled vs base

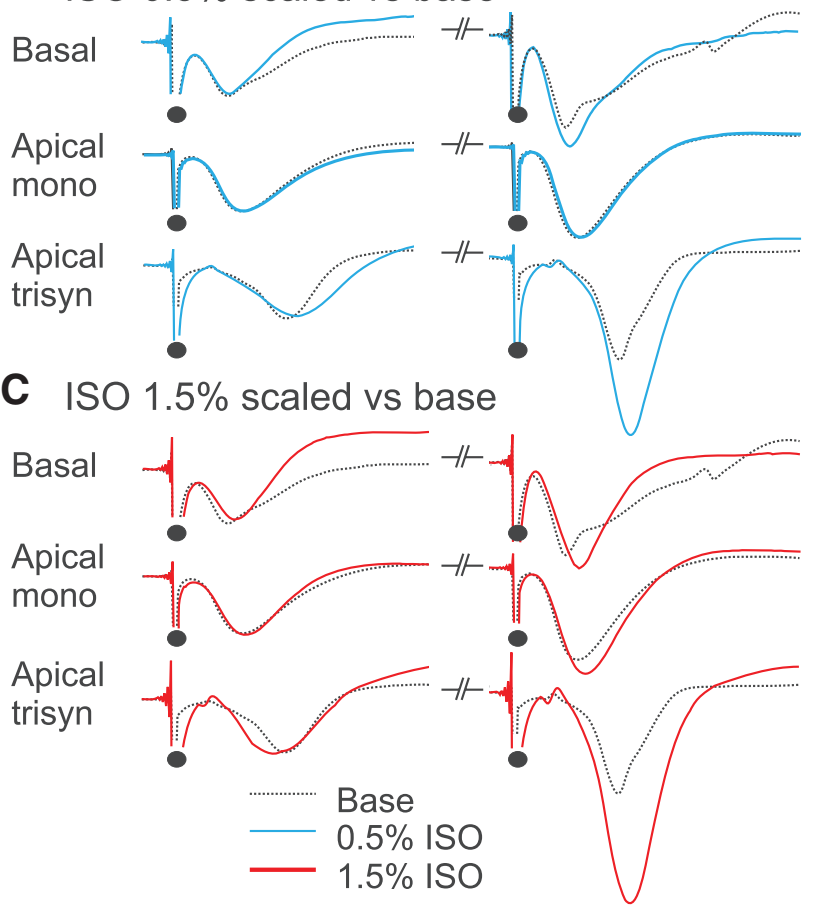

Fig. 5. Paired-pulse facilitation (PPF) of population excitatory postsynaptic potentials (pEPSPs) was enhanced by isoflurane. $(A)$ Average pEPSPs recorded in stratum oriens after oriens stimulation (basal), and in stratum radiatum after radiatum stimulation (apical monosynaptic) or medial perforant path stimulation (apical trisynaptic), for all three conditions: baseline (Base), isoflurane (ISO) 0.5, and 1.5\%; data were from one rat with responses shown in Figs. 2A, 4A, and 4C. $(B)$ Overlay of the pEPSPs for baseline and isoflurane $0.5 \%$ condition, with the first pulse response after isoflurane scaled to match the peak amplitude of baseline. (C) Same as $(B)$, except first pulse response after $1.5 \%$ isoflurane was scaled to match the first pulse baseline. All three stimuli showed enhanced PPF after isoflurane, except no change in apicalmono PPF after $0.5 \%$ isoflurane, and the MPP late (trisynaptic) responses showing the most robust PPF.

not statistically significant at any isoflurane dose tested. The change in spiking with synaptic excitation was shown by plotting the PS1 sink, derived from CSD analysis, with the excitatory apical sink slope E1; both PS1 and E1 were evoked by the first pulse at RAD. For most (four of five) rats, there was no change in the PS1 versus E1 plot after isoflurane, as shown by the scatter plot of three of them before and after
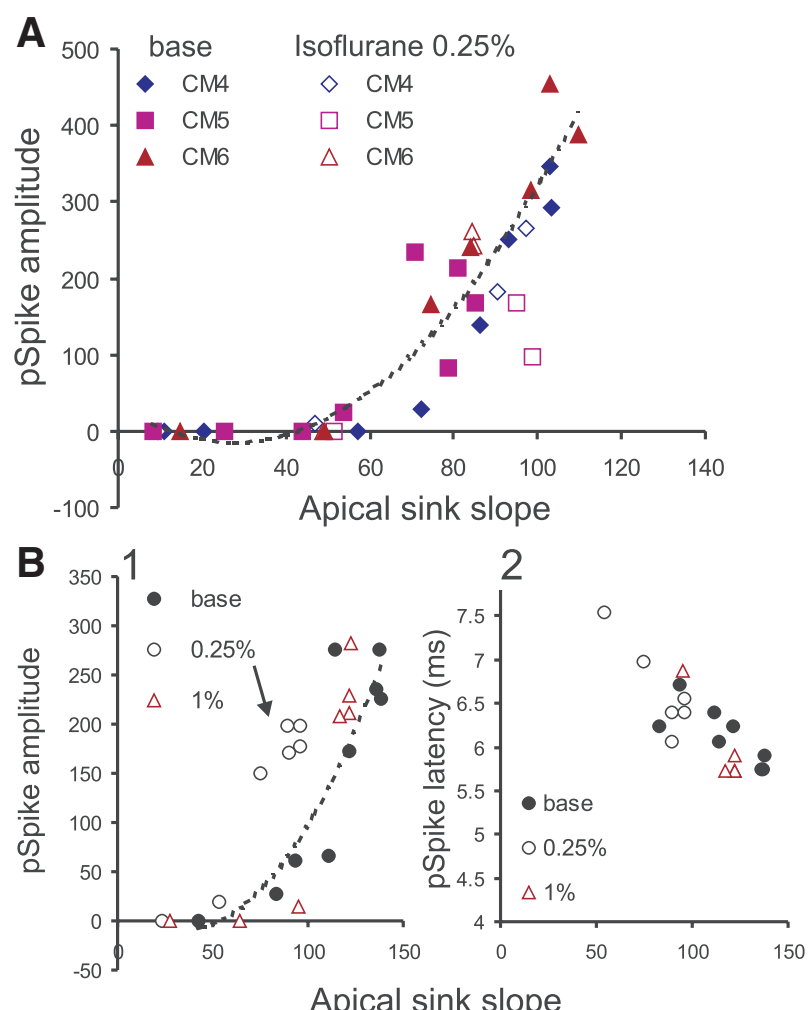

Apical sink slope

Fig. 6. Plot of population spike sink amplitude (PS) versus apical dendritic excitatory sink slope (E) after the first stimulus pulse shows no consistent change after $0.25 \%$ isoflurane. $(A)$ Group data from three rats with PS (y) plotted versus $\mathrm{E}(\mathrm{x})$ during baseline (solid symbols) and after $0.25 \%$ isoflurane (open symbols). Dotted line shows polynomial curve fit $(y=0.064 \times 2$ $-3.58 \mathrm{x}+34.9 ; R^{2}=0.88$ ) of the baseline data of all three rats. (B1) Plots of another rat not included in $(A)$, show an increase in PS amplitude at intermediate $E$ after $0.25 \%$ isoflurane (arrow), above the polynomial curve fit of the baseline data $\left(y=0.074 x^{2}-5.05 x+62.8 ; R^{2}=0.57\right)$. (B2) No consistent change in PS peak latency as a function of $E$ after 0.25 or $1 \%$ isoflurane. PS units in $\mathrm{mV} / \mathrm{mm}^{2}$; $E$ units in $\mathrm{mV} / \mathrm{mm}^{2} / \mathrm{ms}$.

$0.25 \%$ isoflurane (fig. 6A). However, one of five rats did show an increase in PS1 amplitude at intermediate E1 values after $0.25 \%$ isoflurane (arrow at fig. 6B1). A general lack of PS1 versus $\mathrm{E} 1$ was also found at 0.5 to $1.5 \%$ isoflurane doses.

Peak latency of the PS evoked by a constant stimulus intensity showed a consistent increase after greater than or equal to $0.25 \%$ isoflurane dose (data not shown). There was no significant change of PS1 latency in relation to E1 at any dose, as shown by a representative example (fig. 6B2).

\section{Changes in Spontaneous LFPs}

Isoflurane at $0.25 \%$ suppressed power of the LFPs recorded in CA1 RAD during immobility as compared to baseline immobility (fig. 7A), as shown by group-average power spectra (fig. 7B). Selected frequency ranges suppressed by $0.25 \%$ isoflurane were 4 to $30 \mathrm{~Hz}, 50$ to $70 \mathrm{~Hz}$, and 100 to $150 \mathrm{~Hz}$ (fig. 7, B and C). Isoflurane dose at greater than or equal to $0.5 \%$ suppressed power within most of the 0.8 to 
A

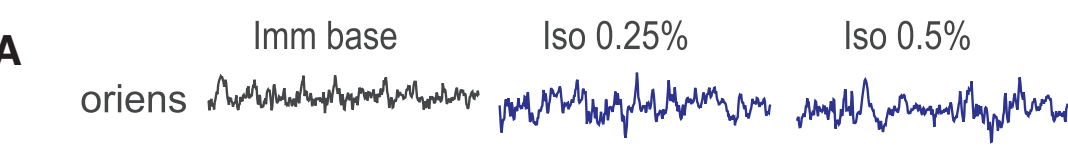

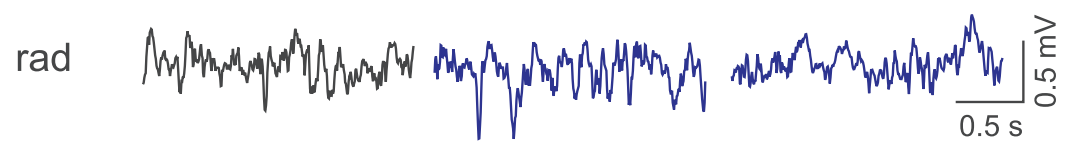

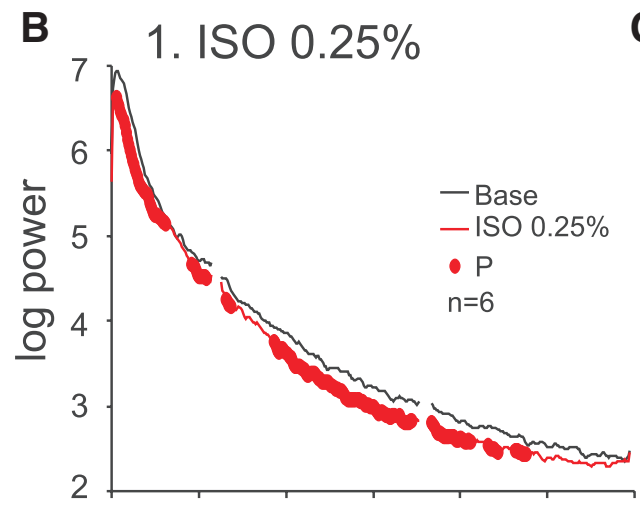

C 1. Str radiatum
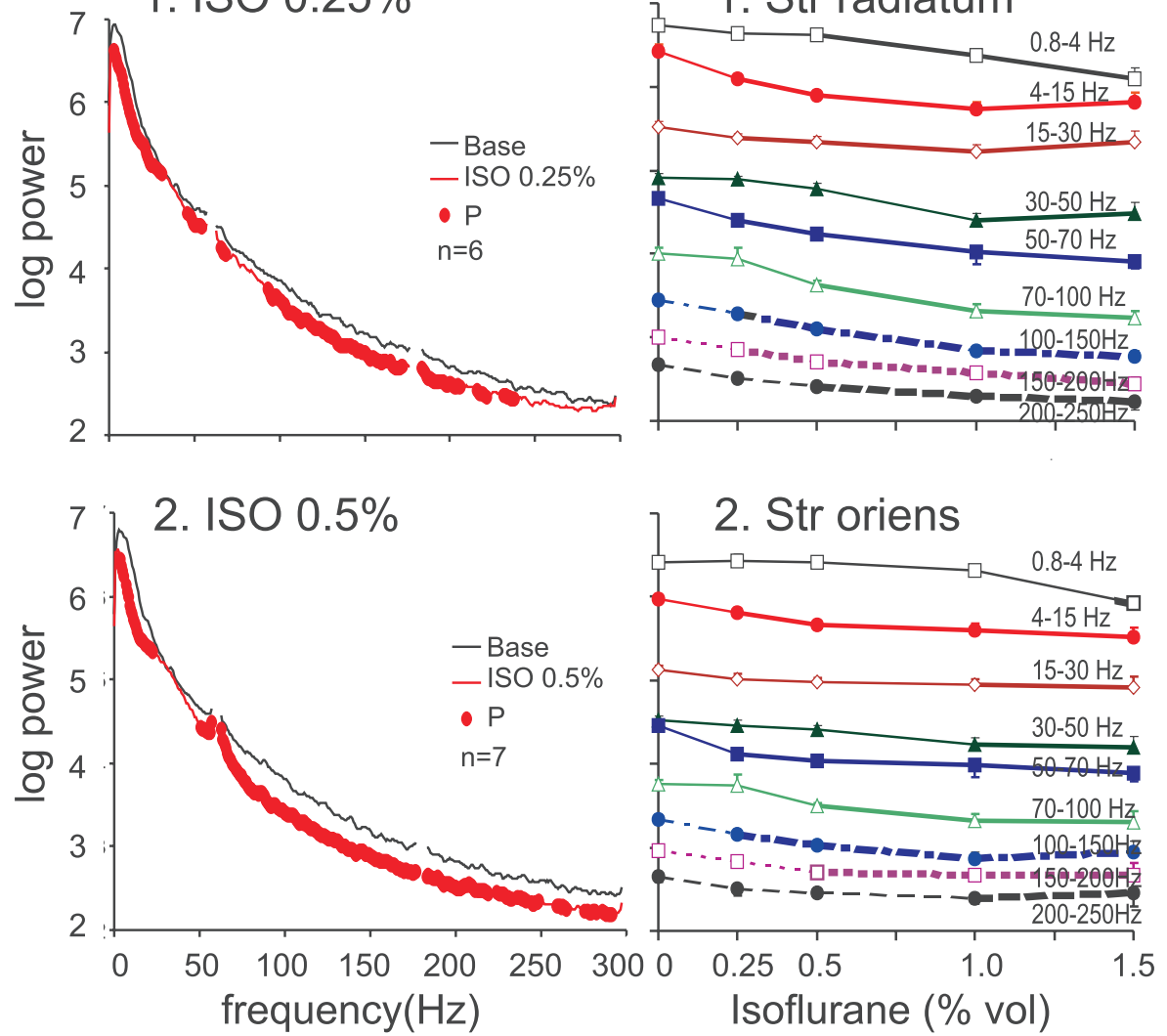

Fig. 7. Spontaneous local field potentials (LFPs) in hippocampal CA1 area were decreased by isoflurane at a wide range of frequency. (A) Representative spontaneous LFPs at stratum oriens and stratum radiatum (rad) during immobility, for baseline and after 0.25 and $0.5 \%$ isoflurane (ISO). (B) Average rad LFP power spectra during baseline compared with during (B1) isoflurane $0.25 \%$ ( $n=6$ rats), or (B2) isoflurane $0.5 \%$ ( $n=7$ rats); 5 or more consecutive time points in the isoflurane spectra that were significantly different from baseline (see Materials and Methods) are labeled with large circles (P). For clarity, line power at 57 to $63 \mathrm{~Hz}$ was digitally removed. Logarithmic power units are $(\mu \mathrm{V})^{2} / \mathrm{Hz} ; 6$ log power units $=1 \mathrm{mV}$ peak amplitude. (C) Group average logarithmic power of spontaneous LFPs for specific frequency ranges as a function of isoflurane dose, recorded at (C1) CA1 stratum (str) radiatum, and (C2) str. oriens. Points that are significantly different from the baseline are connected by a thick line, $P<0.05$, post hoc Newman-Keuls test after a significant two-way repeated measures ANOVA.

$300 \mathrm{~Hz}$ frequency band, except 30 to $50 \mathrm{~Hz}$ power was not suppressed at $0.5 \%$ (fig. 7, B and C). LFPs recorded in OR showed a similar dose dependence on isoflurane, except the power decrease was slightly smaller (fig. 7C).

During baseline, a hippocampal $\Theta$ rhythm was found to accompany walking and REM, with a similar average peak frequency of $-6.8 \mathrm{~Hz}$. The hippocampal $\Theta$ rhythm found during immobility under isoflurane was considered to be associated with REM. During low isoflurane doses $(0.25$ to $0.5 \%$ ), a hippocampal $\Theta$ rhythm was still found during walking and immobility, but its peak frequency was reduced as compared to baseline (table 1). Two-way (behavioral state $\mathrm{x}$ isoflurane dose up to $0.5 \%$ ) randomized block ANOVA showed that the peak hippocampal $\Theta$ frequency did not differ between walking and REM states $(\mathrm{F}[1,4]=1.11, P>0.3$, $\mathrm{n}=5$ rats), but progressively decreased with isoflurane dose $(\mathrm{F}[2,8]=35.1, P<0.001$; table 1$)$. At greater than or equal to $1 \%$ isoflurane, the rat was not capable of spontaneous movements, and the hippocampal LFPs showed irregular slow activity with occasional periods of 3 to $4 \mathrm{~Hz} \Theta$ rhythm.

\section{Low-dose Pentobarbital Responses}

Evoked pEPSPs and LFPs were recorded in seven rats during no-drug baseline and after a single subanesthetic $(10 \mathrm{mg} / \mathrm{kg}$ intraperitoneal) dose of pentobarbital. After pentobarbital, 
Table 1. Isoflurane Dose Effect on Immobile and Walking $\Theta$ Peak Frequency

\begin{tabular}{|c|c|c|c|c|}
\hline Isoflurane \% & Baseline (0\%) & $0.25 \%$ & $0.5 \%$ & $1.5 \%$ \\
\hline Immobile $\Theta$ peak frequency $(\mathrm{Hz})$ & $\begin{array}{c}6.46 \pm 0.53 \\
(n=5)\end{array}$ & $\begin{array}{c}5.70 \pm 0.81^{\text {** }} \\
(\mathrm{n}=5)\end{array}$ & $\begin{array}{c}4.79 \pm 0.49^{\star *} \\
(n=5)\end{array}$ & $\begin{array}{c}4.00 \pm 0.43 \\
(n=3)\end{array}$ \\
\hline Walking $\Theta$ peak frequency $(\mathrm{Hz})$ & $\begin{array}{c}6.81 \pm 0.54 \\
(n=5)\end{array}$ & $\begin{array}{c}4.77 \pm 0.72^{*} \\
(n=5)\end{array}$ & $\begin{array}{c}4.90 \pm 0.38^{\star *} \\
(n=5)\end{array}$ & Not available \\
\hline
\end{tabular}

Mean $\pm \mathrm{SD}$ of the peak $\Theta$ frequency $(\mathrm{Hz})$ recorded in $\mathrm{CA} 1$ stratum radiatum during immobility and walking for baseline and different isoflurane doses. Rats were not capable of walking at 1 to $1.5 \%$ isoflurane.

${ }^{*} P<0.05,{ }^{*} P<0.01$, difference from baseline for the same behavioral state, Newman-Keuls post hoc test after a significant two-way repeated measures ANOVA $(P<0.05)$.

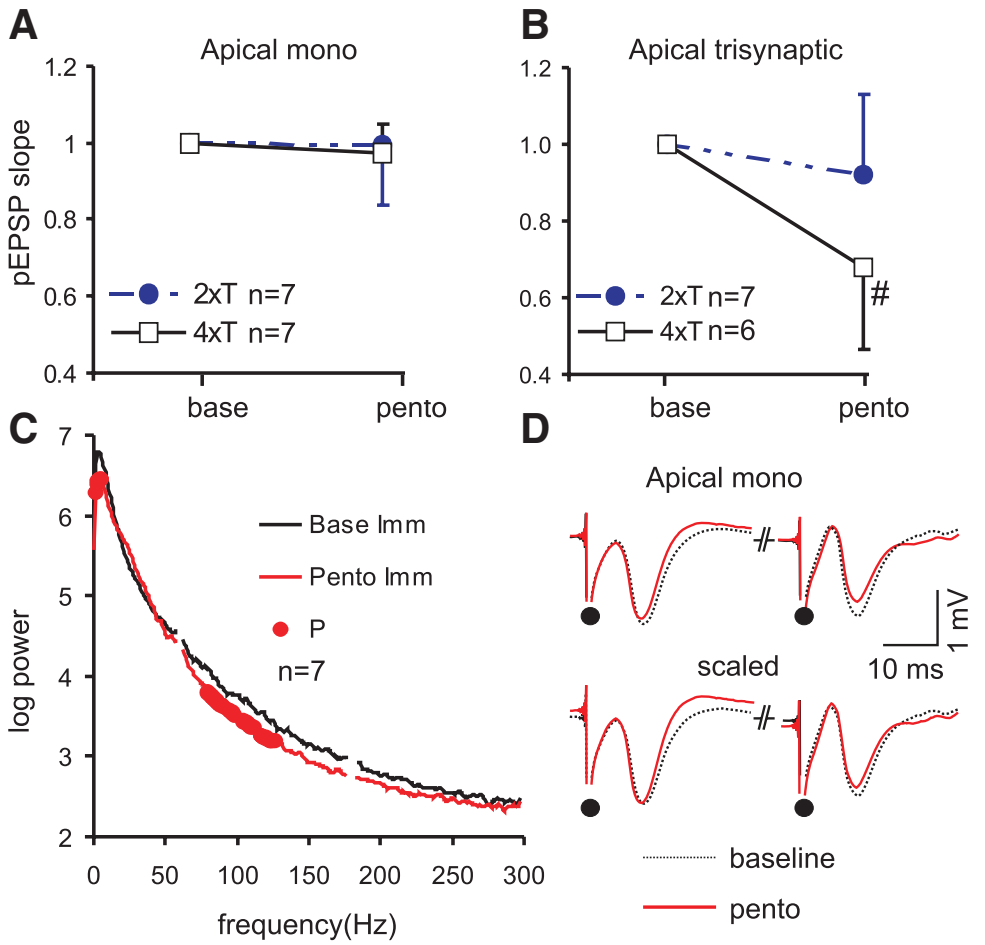

Fig. 8. A subanesthetic dose of pentobarbital $(10 \mathrm{mg} / \mathrm{kg}$ intraperitoneal) disrupted multisynaptic response and hippocampal $\gamma$ frequency local field potentials (LFPs). (A) Pentobarbital $10 \mathrm{mg} / \mathrm{kg}$ intraperitoneal did not significantly affect monosynaptic apical dendritic pEPSP (after stratum radiatum stimulation), but significantly decreased the $(B)$ multisynaptic apical pEPSP after medial perforant path stimulation (Apical trisynaptic) at $4 \mathrm{x}$ threshold stimulus intensity $(4 \mathrm{xT})$. Responses were normalized to unity at baseline, and shown as mean $\pm \mathrm{SD}$. $\# P<0.05$ compared to baseline. In $A-B$, two rats were recorded with wire electrodes, and the remaining rats with a 16-channel probe. $(C)$ Average logarithmic power spectra of spontaneous LFPs at CA1 stratum radiatum during immobility (Imm), before (baseline), and after $10 \mathrm{mg} / \mathrm{kg}$ intraperitoneal pentobarbital (pento, $\mathrm{n}=7 \mathrm{rats}$ ). Significant differences (paired Wilcoxon for $\geq 5$ consecutive time points) between baseline and pentobarbital spectra are labeled by a large circle $(P)$. Power at 57 to $63 \mathrm{~Hz}$ was digitally removed. $(D)$ Paired-pulse facilitation of pEPSPs, at 50-ms interpulse interval, was reduced after pentobarbital, shown by a representative recording of apical pEPSPs after stratum radiatum stimulation.

the pEPSP after OR stimulation (not shown) and RAD stimulation (fig. 8A) were not significantly changed from baseline, but the trisynaptic CA1 pEPSP at $4 \mathrm{x}$ threshold intensity was significantly reduced (fig. 8B). Group average spectra of LFP power at RAD shows that, as compared to baseline, the power at $\Delta(1$ to $4 \mathrm{~Hz})$ and high $\gamma$ frequency (78 to $125 \mathrm{~Hz}$ ) were significantly decreased after pentobarbital injection (fig. 8C). After pentobarbital, apical pEPSPs showed paired-pulse depression (decreased E2/E1 ratio as shown in fig. 8D), as compared to increased PPF after isoflurane.

\section{Discussion}

\section{Excitatory Synaptic Transmission Was Decreased by Low-dose Isoflurane}

We reported that hippocampal synaptic transmission in freely moving rats was dose-dependently suppressed by isoflurane. The excitatory synapse on the basal dendrites of CA1 pyramidal cells, as compared to that on the apical dendrites, was suppressed by the lowest dose of isoflurane used $(0.25 \%)$. The multisynaptic pathway from the EC to CA1 was also suppressed by $0.25 \%$ isoflurane. Spontaneous LFP 
power across a wide band of frequency $(0.8$ to $300 \mathrm{~Hz})$ also showed a dose-dependent suppression by isoflurane.

The monosynaptic apical dendritic pEPSP (evoked by RAD stimulation) decreased in a graded manner with isoflurane dose and showed a statistically significant decline at greater than or equal to $1 \%$ isoflurane (fig. $3 \mathrm{~B}$ ). This isoflurane dose response was similar to the $\mathrm{PEPSP}^{10}$ or the intracellularly recorded EPSPs in CA1 in vitro. ${ }^{7,8}$ The CA1 basal dendritic pEPSP (evoked by OR stimulation) in vivo demonstrated an exquisite sensitivity to isoflurane, being suppressed by $0.25 \%$ isoflurane (fig. 3A). Sensitivity of the OR-evoked pEPSP to $0.5 \%$ isoflurane was reported in vitro.

The multisynaptic MPP-DG-CA3-CA1 response (triE) and an OR-evoked recurrent excitation of CA1 (fig. 2A) were suppressed by 0.25 to $0.5 \%$ isoflurane. The latter isoflurane dose did not significantly alter the MPP-evoked PS in the DG or the RAD-evoked apical dendritic excitation in CA1, but transmission in CA3 had not been studied. Isoflurane may suppress multisynaptic transmission readily because small decrease in excitatory transmission and spike excitability at one synapse may accumulate across multiple synapses. Low doses of isoflurane were shown to enhance GABAergic inhibition ${ }^{13-15}$ and suppress neuronal firing of both pyramidal and inhibitory neurons. ${ }^{38}$ However, $\gamma$-aminobutyric acid type $A\left(G_{A B A}\right)$ receptor antagonist bicuculline, which blocked tonic and evoked $\mathrm{GABA}_{\mathrm{A}}$ receptor-mediated inhibition, had no consistent effect on the rising phase of monosynaptic EPSPs in CA1 neurons in vitro. ${ }^{39}$ An effect of tonic ${ }^{15}$ or late ${ }^{13}$ GABAergic inhibition on triE in vivo remains to be shown. Still, it may be fair to assume isoflurane's suppression of triE results from cumulative changes in excitation and inhibition along the multisynaptic pathway.

PPF was found for basal or apical dendritic pEPSPs evoked at low-stimulus intensities. An isoflurane dose that decreased the first-pulse pEPSP also significantly increased PPF. Thus, the isoflurane sensitivity of the PPF of each pathway paralleled that of the first-pulse pEPSP. PPF was more robust for pEPSPs after OR than RAD stimulation (with the same $x$ threshold intensity), and large for the triE after MPP stimulation (fig. 5). PPF at a single synapse is associated with release probability, and a high release probability (strong synapse) is associated with small PPF. ${ }^{40}$ The change in pEPSPs after isoflurane can be explained by a decrease in neurotransmitter release, ${ }^{10}$ possibly selective at glutamatergic terminals. ${ }^{12}$ Isoflurane $(-0.5 \mathrm{MAC})$ was shown to decrease exocytosis of synaptic vesicles at glutamatergic terminals in vitro. ${ }^{41}$

We did not find a consistent isoflurane-induced change in the relation between single-pulse PS amplitude and excitatory strength E1, measured by slope of the pEPSP or excitatory sink in vivo (fig. 6). Spiking in CA1 neurons after intracellular current injection was not sensitive to 0.5 to $3 \%$ isoflurane, ${ }^{8}$ suggesting that spike excitability had low sensitivity to isoflurane. The lack of a consistent increase in single-pulse PS1 versus E1 after $0.25 \%$ isoflurane (fig. 6A) appears to be different from the conclusion of increased spike excitability induced by paired-pulse stimulation after $0.5 \%$ isoflurane. ${ }^{9}$
Our results of a significant reduction of glutamatergic excitation in vivo differ from a previous study, ${ }^{23}$ which concluded that isoflurane had no clear effects on hippocampal pEPSP and PS after commissural stimulation. However, the latter study ${ }^{23}$ did not include a preanesthetic baseline, and effects after isoflurane were compared with those after urethane anesthesia. The current study also differs from another in vivo study that reported 0.38 to $1.4 \%$ volume isoflurane caused a leftward shift of the Schaffer-collaterals evoked PS versus $\mathrm{pEPSP}$ curve,${ }^{24}$ with both PS and pEPSP recorded from the CA1 pyramidal cell layer. Since the excitatory synapses are on the dendrites, optimal recording of pEPSP, or excitatory sink, would be at the apical dendritic layer after RAD stimulation, or the basal dendritic layer after OR stimulation. In our multichannel recordings, we observed that $1.5 \%$ isoflurane decreased the positive pEPSP near the soma $25 \%$ more than the negative pEPSP at the apical dendrites, possibly because of overlap with spike prepotential and inhibitory conductance near the cell body.

\section{Spontaneous Hippocampal Field Potentials Were Sensitive to Low-dose Isoflurane}

We showed that the spontaneous hippocampal LFP power was decreased by isoflurane dose of greater than or equal to $0.25 \%$. The most sensitive frequency bands appear to be 4 to $15 \mathrm{~Hz}$ and 50 to $150 \mathrm{~Hz}$ (fig. 7), consistent with our previous studies. ${ }^{42-44}$ Similarly, Hudetz et al. ${ }^{45}$ showed that 70 to $140 \mathrm{~Hz}$ power of spontaneous LFPs in the hippocampus, visual and frontal cortices were decreased by greater than or equal to $0.4 \%$ isoflurane. The current study decreased the sensitivity of hippocampal LFPs to $0.25 \%$ isoflurane, and similar sensitivity was found for LFPs recorded in OR and $\mathrm{RAD}$, except the drop in LFP power with isoflurane dose was somewhat smaller in OR than RAD (fig. 7C).

We found that the peak frequency of the walking hippocampal $\Theta$ rhythm decreased with isoflurane dose $(0.25$ to $0.5 \%$ ), as was reported previously. ${ }^{16}$ In addition, under low-dose isoflurane, a $\Theta$ rhythm that occurred during immobility, tentatively identified as REM, showed a similar peak frequency as that during walking. Thus, isoflurane's effect in slowing $\Theta$ frequency was independent of behavioral states. This effect was likely exerted outside of the hippocampus, possibly on the medial septum and supramammillary area..$^{25,26}$

Sensitivity of $\gamma$ waves to low isoflurane doses is consistent with anesthestic sensitivity of a multisynaptic network. $\gamma$ activity in the hippocampal and neocortical cortex in vivo is generated by perisomatic parvalbumin-positive interneurons interacting with principal cells (fig. 9A), ${ }^{46-48}$ although purely GABAergic networks of interneurons could generate $\gamma$ oscillations as well. ${ }^{49}$ LFPs recorded in CA1, including $\gamma$ activity, may be intrinsically generated, or driven by similar generating networks in $\mathrm{CA} 3$ and $\mathrm{EC} .{ }^{27,28}$ The power of spontaneous hippocampal LFPs $(0$ to $100 \mathrm{~Hz})$ was strongly attenuated by non-N-methyl-D-aspartate receptor antagonists, ${ }^{50}$ supporting the concept that hippocampal LFPs are driven by 

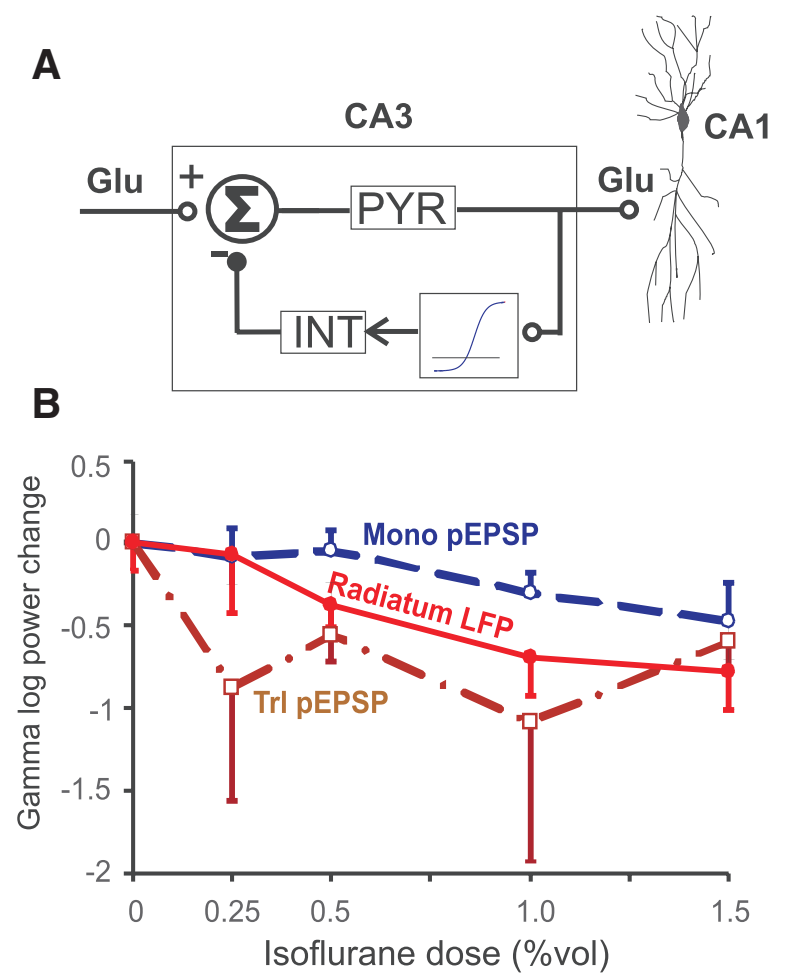

Fig. 9. Glutamatergic involvement in spontaneous local field potential (LFP) generation in hippocampal CA1. (A) Schematic diagram of glutamatergic driving of $\gamma$ rhythm in CA1 (adapted from Leung ${ }^{46}$ ). Apical dendritic $\gamma$ activity in CA1 is driven by CA3 pyramidal cells (PYR) being recurrently inhibited by somatic interneurons (INT). CA3 receives glutamatergic (Glu) inputs from the dentate gyrus and entorhinal cortex. $(B)$ Mean logarithmic power change from baseline (normalized to 0 ) with isoflurane dose (\% vol) of the 70 to $100 \mathrm{~Hz} \gamma$-band LFPs recorded in stratum radiatum, compared to the power (amplitude square) change of the normalized population excitatory postsynaptic potentials evoked by apical dendritic monosynaptic (mono pEPSP) or trisynaptic excitation (tri pEPSP). Error bar is one SD. Mono pEPSP power change is similar to radiatum LFP power change at $0.25 \%$ dose while tri-pEPSP power change is similar to radiatum LFP power change at 0.5 to $1.5 \%$ dose.

non-N-methyl-D-aspartate glutamatergic inputs. Using 70 to $100 \mathrm{~Hz}$ (RAD-recorded) LFP power to represent $\gamma$, the decrease of $\gamma$ power with 0.5 to $1.5 \%$ isoflurane dose was found to be similar to the decrease in power (square amplitude) of the trisynaptic apical pEPSPs (fig. 9B). This suggests that the main cause of LFP $\gamma$ power decrease with high isoflurane dose is a reduction in glutamatergic synaptic transmission.

\section{Isoflurane Dose Induces Synaptic and Behavioral Deficits}

Isoflurane of $0.28 \%$ induced deficits in a context-dependent fear conditioning in rats, ${ }^{3}$ a task that depends on the hippocampus. ${ }^{19,20}$ The results here showed that $0.25 \%$ isoflurane disrupted hippocampal $\Theta$ and $\gamma$ activity. More direct evidence of interference of hippocampal function is shown by a strong impairment of the multisynaptic transmission from the EC to CA1, which has been suggested as a main neural circuit responsible for hippocampal memory. ${ }^{21}$
Sodium pentobarbital, at a subanesthetic dose $(10 \mathrm{mg} / \mathrm{kg}$ intraperitoneal) that did not induce loss of righting reflex, disrupted multisynaptic transmission without significantly changing monosynaptic apical or basal dendritic responses. Pentobarbital is known to augment $\mathrm{GABA}_{\mathrm{A}}$ receptormediated inhibition, ${ }^{14,51}$ and triE transmission may decline because of difficulty to generate action potentials at multiple areas along the trisynaptic pathway. Pentobarbital's effect in decreasing PPF of the pEPSPs (fig. 8D) is consistent with an enhanced inhibition that suppressed the second pulse pEPSP. $^{52}$ In the presence of a $\mathrm{GABA}_{\mathrm{A}}$ receptor antagonist, pentobarbital increased PPF of the pEPSPs. ${ }^{14}$ Spontaneous LFPs in the high $\gamma$ frequency (78 to $125 \mathrm{~Hz}$ ) range were also suppressed by low-dose pentobarbital.

\section{Limitations of the Current Study}

The current study on field potentials emphasized glutamatergic transmission in vivo and did not fully reveal inhibitory or other ionic events that may be affected by isoflurane. Results in vitro showed that amnestic doses of isoflurane $(-0.25$ MAC) significantly increased tonic GABAergic currents ${ }^{15}$ or prolonged evoked inhibitory postsynaptic currents in hippocampal pyramidal cells. ${ }^{13}$ The current data do not fully reveal the inhibitory changes induced by isoflurane. In addition, population events such as PS do not reveal excitability of the different types of cells, and isoflurane's action on specific types of interneurons or on CA3 cells is not known. Cellular mechanisms of isoflurane's action, such as a decrease in glutamatergic release, could only be inferred in the current study, and await direct verification with other techniques.

Isoflurane dose response may differ in different areas of the brain, ${ }^{1,53}$ and the current data on the hippocampus do not explain the loss of righting or pain responses. We propose a working hypothesis that isoflurane's reduction of glutamatergic transmission in the hippocampus accounts for some of the amnestic effects of low-dose isoflurane. While the hypothesis is intuitive, it is tentative in view of the multiple effects of isoflurane on the brain. Furthermore, memory function was not studied in the animals recorded in the current study. In order to validate the working hypothesis, future experiments will need to directly reverse isoflurane's glutamatergic effects selectively in the hippocampus.

\section{Research Support}

This research was supported by the Canadian Institutes of Health Research (grant No. MOP-15685), Ottawa, Ontario, Canada, and the Natural Sciences and Engineering Research Council (grant No. 1037-2008), Ottawa, Ontario, Canada.

\section{Competing Interests}

The authors declare no competing interests.

\section{Correspondence}

Address correspondence to Dr. Leung: Department of Physiology and Pharmacology, The University of Western On- 
tario, London, Ontario N6A5C1, Canada. sleung@uwo.ca Information on purchasing reprints may be found at www. anesthesiology.org or on the masthead page at the beginning of this issue. Anesthesiology's articles are made freely accessible to all readers, for personal use only, 6 months from the cover date of the issue.

\section{References}

1. Antognini JF, Carstens E: In vivo characterization of clinical anaesthesia and its components. Br J Anaesth 2002; 89:156-66

2. Campagna JA, Miller KW, Forman SA: Mechanisms of actions of inhaled anesthetics. N Engl J Med 2003; 348:2110-24

3. Dutton RC, Maurer AJ, Sonner JM, Fanselow MS, Laster MJ, Eger EI II: The concentration of isoflurane required to suppress learning depends on the type of learning. ANESTHESIOLOGY 2001; 94:514-9

4. Dwyer R, Bennett HL, Eger EI II, Heilbron D: Effects of isoflurane and nitrous oxide in subanesthetic concentrations on memory and responsiveness in volunteers. ANESTHESIOLOGY 1992; 77;888-98

5. Franks NP: General anaesthesia: From molecular targets to neuronal pathways of sleep and arousal. Nat Rev Neurosci 2008; 9:370-86

6. Richards CD, White AE: The actions of volatile anaesthetics on synaptic transmission in the dentate gyrus. J Physiol 1975; 252:241-57

7. Berg-Johnsen J, Langmoen IA: The effect of isoflurane on excitatory synaptic transmission in the rat hippocampus. Acta Anaesthesiol Scand 1992; 36:350-5

8. Miu P, Puil E: Isoflurane-induced impairment of synaptic transmission in hippocampal neurons. Exp Brain Res 1989; 75:354-60

9. MacIver MB, Roth SH: Inhalation anaesthetics exhibit pathway-specific and differential actions on hippocampal synaptic responses in vitro. Br J Anaesth 1988; 60:680-91

10. Maclver MB, Mikulec AA, Amagasu SM, Monroe FA: Volatile anesthetics depress glutamate transmission via presynaptic actions. ANESTHESIOLOGY 1996; 85:823-34

11. Pittson S, Himmel AM, MacIver MB: Multiple synaptic and membrane sites of anesthetic action in the CA1 region of rat hippocampal slices. BMC Neurosci 2004; 5:52

12. Zimin PI, Woods CB, Quintana A, Ramirez JM, Morgan PG, Sedensky MM: Glutamatergic neurotransmission links sensitivity to volatile anesthetics with mitochondrial function. Curr Biol 2016; 26:2194-201

13. Dai S, Perouansky M, Pearce RA: Isoflurane enhances both fast and slow synaptic inhibition in the hippocampus at amnestic concentrations. ANESTHESIOLOGY 2012; 116:816-23

14. MacIver MB: Anesthetic agent-specific effects on synaptic inhibition. Anesth Analg 2014; 119:558-69

15. Caraiscos VB, Newell JG, You-Ten KE, Elliott EM, Rosahl TW, Wafford KA, MacDonald JF, Orser BA: Selective enhancement of tonic GABAergic inhibition in murine hippocampal neurons by low concentrations of the volatile anesthetic isoflurane. J Neurosci 2004; 24:8454-8

16. Perouansky M, Rau V, Ford T, Oh SI, Perkins M, Eger EI II, Pearce RA: Slowing of the hippocampal $\theta$ rhythm correlates with anesthetic-induced amnesia. ANESTHESIOLOGY 2010; 113:1299-309

17. Andersen P, Bliss TV, Skrede KK: Lamellar organization of hippocampal pathways. Exp Brain Res 1971; 13:222-38

18. Lopes da Silva FH, Witter MP, Boeijinga PH, Lohman AH: Anatomic organization and physiology of the limbic cortex. Physiol Rev 1990; 70:453-511

19. Lee I, Kesner RP: Differential contributions of dorsal hippocampal subregions to memory acquisition and retrieval in contextual fear-conditioning. Hippocampus 2004; 14:301-10

20. White PF, Johnston RR, Eger EI II: Determination of anesthetic requirement in rats. ANESTHESIOLOGY 1974; 40:52-7
21. Zola-Morgan S, Squire LR, Amaral DG: Human amnesia and the medial temporal region: Enduring memory impairment following a bilateral lesion limited to field CA1 of the hippocampus. J Neurosci 1986; 6:2950-67

22. Klausberger T, Somogyi P: Neuronal diversity and temporal dynamics: The unity of hippocampal circuit operations. Science 2008; 321:53-7

23. Pearce RA, Stringer JL, Lothman EW: Effect of volatile anesthetics on synaptic transmission in the rat hippocampus. ANESTHESIOLOGY 1989; 71:591-8

24. Tachibana K, Takita K, Hashimoto T, Matsumoto M, Yoshioka $\mathrm{M}$, Morimoto $\mathrm{Y}$ : Isoflurane bidirectionally modulates the paired-pulse responses in the rat hippocampal CA1 field in vivo. Anesth Analg 2007; 105:1006-11

25. Bland $\mathrm{BH}$, Colom LV: Extrinsic and intrinsic properties underlying oscillation and synchrony in limbic cortex. Prog Neurobiol 1993; 41:157-208

26. Buzsáki G: Theta oscillations in the hippocampus. Neuron 2002; 33:325-40

27. Colgin LL, Denninger T, Fyhn M, Hafting T, Bonnevie T, Jensen $\mathrm{O}$, Moser MB, Moser EI: Frequency of gamma oscillations routes flow of information in the hippocampus. Nature 2009; 462:353-7

28. Csicsvari J, Jamieson B, Wise KD, Buzsáki G: Mechanisms of gamma oscillations in the hippocampus of the behaving rat. Neuron 2003; 37:311-22

29. Leung LS: Generation of theta and gamma rhythms in the hippocampus. Neurosci Biobehav Rev 1998; 22:275-90

30. Wolansky T, Clement EA, Peters SR, Palczak MA, Dickson CT: Hippocampal slow oscillation: A novel EEG state and its coordination with ongoing neocortical activity. J Neurosci 2006; 26:6213-29

31. Paxinos G, Watson C: The Rat Brain in Stereotaxic Coordinates, 4th edition. New York, Academic Press, 1998

32. Kloosterman F, Peloquin P, Leung LS: Apical and basal orthodromic population spikes in hippocampal CA1 in vivo show different origins and patterns of propagation. J Neurophysiol 2001; 86:2435-44

33. Fung TK, Law CS, Leung LS: Associative spike timingdependent potentiation of the basal dendritic excitatory synapses in the hippocampus in vivo. J Neurophysiol 2016; 115:3264-74

34. Leung LS, Péloquin P: Cholinergic modulation differs between basal and apical dendritic excitation of hippocampal CA1 pyramidal cells. Cereb Cortex 2010; 20:1865-77

35. Leung LS: Field potential generation and current source density analysis. In: Vertes RP, Stackman RW (Eds): Electrophysiological Recording Techniques, NeuroMethods 2010; 15:1-26, Clifton, NJ, Humana

36. Leung LS, Lopes da Silva FH, Wadman WJ: Spectral characteristics of the hippocampal EEG in the freely moving rat. Electroencephalogr Clin Neurophysiol 1982; 54:203-19

37. Wu K, Leung LS: Increased dendritic excitability in hippocampal ca1 in vivo in the kainic acid model of temporal lobe epilepsy: A study using current source density analysis. Neuroscience 2003; 116:599-616

38. Bieda MC, MacIver MB: Major role for tonic GABAA conductances in anesthetic suppression of intrinsic neuronal excitability. J Neurophysiol 2004; 92:1658-67

39. Leung LS, Fu XW: Factors affecting paired-pulse facilitation in hippocampal CA1 neurons in vitro. Brain Res 1994; 650:75-84

40. Zucker RS: Short-term synaptic plasticity. Annu Rev Neurosci 1989; 12:13-31

41. Baumgart JP, Zhou ZY, Hara M, Cook DC, Hoppa MB, Ryan TA, Hemmings HC Jr: Isoflurane inhibits synaptic vesicle exocytosis through reduced $\mathrm{Ca} 2+$ influx, not $\mathrm{Ca} 2+-$ exocytosis coupling. Proc Natl Acad Sci USA 2015; 112:11959-64

42. Ma J, Shen B, Stewart LS, Herrick IA, Leung LS: The septohippocampal system participates in general anesthesia. J Neurosci 2002; 22:RC200 
43. Tai SK, Ma J, Leung LS: Medial septal cholinergic neurons modulate isoflurane anesthesia. ANESTHESIOLOGY 2014; 120:392-402

44. Leung LS, Luo T, Ma J, Herrick I: Brain areas that influence general anesthesia. Prog Neurobiol 2014; 122:24-44

45. Hudetz AG, Vizuete JA, Pillay S: Differential effects of isoflurane on high-frequency and low-frequency $\gamma$ oscillations in the cerebral cortex and hippocampus in freely moving rats. ANESTHESIOLOGY 2011; 114:588-95

46. Leung LS: Nonlinear feedback model of neuronal populations in hippocampal CAl region. J Neurophysiol 1982; 47:845-68

47. Mann EO, Radcliffe CA, Paulsen O: Hippocampal gammafrequency oscillations: From interneurones to pyramidal cells, and back. J Physiol 2005; 562(Pt 1):55-63

48. Sohal VS, Zhang F, Yizhar O, Deisseroth K: Parvalbumin neurons and gamma rhythms enhance cortical circuit performance. Nature 2009; 459:698-702
49. Jefferys JG, Traub RD, Whittington MA: Neuronal networks for induced ' $40 \mathrm{~Hz}$ ' rhythms. Trends Neurosci 1996; 19:202-8

50. Leung LS, Shen B: Glutamatergic synaptic transmission participates in generating the hippocampal EEG. Hippocampus 2004; 14:510-25

51. Leung LS: Differential effects of pentobarbital and ether on the synaptic transmission of the hippocampal CA1 region in the rat. Electroencephalogr Clin Neurophysiol 1981; 51:291-305

52. Leung LS, Peloquin P, Canning KJ: Paired-pulse depression of excitatory postsynaptic current sinks in hippocampal CA1 in vivo. Hippocampus 2008; 18:1008-20

53. MacIver MB, Bland $\mathrm{BH}$ : Chaos analysis of EEG during isoflurane-induced loss of righting in rats. Front Syst Neurosci 2014; 8:203 